\title{
Avaliação da profilaxia do tromboembolismo venoso em hospital de grande porte
}

\section{Evaluation of venous thromboembolism prophylaxis in a high complexity hospital}

\author{
João luiz de Aquino Carneiro, ECBC-ES; Gabriel Pelegrineti Targueta²; Lucas Oliveira Marino³
}

RE S U M O

\begin{abstract}
Objetivo: Avaliar a adequação da tromboprofilaxia em um hospital de grande porte em Vitória-ES, analisando possíveis preditores de aplicação de conduta inadequada. Métodos: Trata-se de um estudo de corte transversal realizado através de análise de prontuários. Os pacientes analisados estiveram internados no hospital durante o ano de 2007, e tiveram seu risco de tromboembolismo venoso estratificado segundo a $8^{\text {a }}$ Diretriz para Profilaxia do TEV do American College of Chest Physicians ( $8^{\circ}$ ACCP). A adequação da tromboprofilaxia foi determinada de acordo com a concordância entre a conduta instituída e a conduta preconizada nas diretrizes. Foram utilizados os softwares Epilnfo 3.4.3 e SPSS 13.0. Resultados: Em 47\% dos pacientes a tromboprofilaxia foi inadequada, sendo a não prescrição da medicação indicada o principal motivo (33\%). Não houve diferença estatisticamente significante quando comparadas as taxas de inadequação da tromboprofilaxia entre pacientes clínicos e cirúrgicos, ou, entre pacientes internados em enfermaria e UTI. O número de fatores de risco para TEV foi inversamente proporcional à taxa de inadequação $(p<0,05)$, assim como a faixa etária do paciente e a duração da internação $(p<0,05)$. Conclusão: Os resultados obtidos apontam para níveis alarmantes de inadequação da tromboprofilaxia, o que evidencia a necessidade de programas de educação continuada no assunto para toda a equipe assistente.
\end{abstract}

Descritores: Tromboembolismo venoso. Profilaxia. Fatores de risco. Heparina.

\section{INTRODUÇÃO}

A trombose venosa profunda e o tromboembolismo pulmonar são dois espectros de uma mesma doença definida pelo termo Tromboembolismo Venoso (TEV). Tratase de uma condição potencialmente grave, estando associada a $5-10 \%$ das mortes em pacientes hospitalizados ${ }^{1}$. Sem profilaxia adequada, a incidência de TEV durante internação hospitalar chega a níveis tão altos quanto 10$40 \%$ entre pacientes clínicos e cirúrgicos e $40-60 \%$ após grandes cirurgias ortopédicas ${ }^{2,3}$. A prevenção primária é, portanto, essencial, quando se considera morbidade e custos hospitalares-8.

No estudo de Kakkar et al. ${ }^{9}$, detectou-se que $50 \%$ ou mais dos casos de TVP foram subclínicos, apontando para a pouca confiança do exame clínico no diagnóstico desta entidade. Somado a isto, o tromboembolismo venoso se mostra como a principal causa de morte súbita e evitável em leitos hospitalares ${ }^{4}$. Dessa forma, a profilaxia da TVP ganhou destaque no cenário hospitalar, tornando-se bastante superior, inclu- sive em custo-efetividade, comparada ao seu tratamento $6,10-12$

Três grandes ensaios clínicos (MEDENOX, PREVENT e ARTEMIS ${ }^{13-15}$ ) evidenciaram reduções de até $63 \%$ do risco relativo de TEV através do uso de profilaxia medicamentosa durante a internação. O que se observa na prática clínica diária, entretanto, é que, a despeito dos avanços no desenvolvimento de drogas anticoagulantes e de métodos mecânicos de prevenção do TEV, esses meios são ainda subutilizados pelo médico-assistente, resultando em risco adicional inaceitável para muitos pacientes $^{16-18}$. Em dois estudos brasileiros ${ }^{4,19}$, detectaram-se taxas altas de inadequação da tromboprofilaxia, atingindo $29 \%$ em pacientes submetidos a intervenções cirurgicas de altíssimo risco para TEV ${ }^{19}$ e até $77 \%$ em pacientes clínicos ${ }^{4}$

O presente estudo tem por objetivo avaliar a adequação da tromboprofilaxia em um hospital de grande porte na cidade de Vitória-ES, o qual realiza internações clínicas e cirurgias de grande e pequeno porte.

Trabalho realizado no Centro Integrado de Atenção à Saúde - CIAS - Vitória - ES - Brasil.

1. Membro Titular Emérito do Colégio Brasileiro de Cirurgiões; Professor Livre Docente Emérito do Departamento de Cirurgia da Universidade Federal do Espírito Santo (UFES); 2. Médico Residente de Clínica Médica da Universidade Federal de São Paulo (UNIFESP); 3. Acadêmico do Curso de Medicina da Universidade Federal do Espírito Santo (UFES). 


\section{MÉTODOS}

Foi realizado um estudo transversal de pacientes internados, abordando as especialidades Clínica Médica, Ortopedia, Clínica Cirúrgica e Unidade de Terapia Intensiva (UTI). Os pacientes foram divididos em duas classes: pacientes internados para tratamento clínico e para tratamento cirúrgico, sendo paciente cirúrgico todo aquele que fora submetido a algum procedimento cirúrgico na internação analisada. Não houve a aplicação de um termo de consentimento livre e esclarecido, pois não ocorreram entrevistas nem contato direto com os pacientes. As análises foram realizadas através do exame do prontuário médico, sendo que a identificação dos pacientes foi totalmente preservada durante a verificação desses prontuários.

Foram analisados no total 247 prontuários de pacientes, 97 correspondendo a pacientes clínicos e 150 cirúrgicos, internados em enfermarias ou em UTI no Centro Integrado de Atenção à Saúde, em Vitória - ES, no período compreendido entre janeiro e dezembro de 2007, escolhidos aleatoriamente. A randomização foi feita a partir de uma lista de todos os pacientes internados no período, selecionando-se múltiplos de 8. Quando um paciente selecionado portava critérios de exclusão, o próximo múltiplo de 8 na listagem era selecionado.

Foram critérios de inclusão a permanência por pelo menos 48 horas no hospital e presença de dados necessários no registro do prontuário. Foram excluídos pacientes pediátricos e os pacientes cujo motivo da internação era TEV.

A análise da indicação de tromboprofilaxia foi baseada em fatores de risco, como preconizado pela $8^{a}$ Diretriz para Profilaxia de TEV do American College of Chest Physicians ( $8^{\circ} \mathrm{ACCP}$ ). Para pacientes cirúrgicos, são considerados de alto risco aqueles submetidos à cirurgia de altíssimo risco para TEV (artroplastia de joelho ou quadril, fratura de quadril, cirurgia oncológica, trauma raquimedular ou politrauma), ou, com idade superior a 60 anos ou entre 40-60 anos na presença de fatores de risco. Pacientes cirúrgicos de risco intermediário para TEV, são aqueles com idade entre 40-60 anos sem fatores de risco, ou, com ida- de menor que 40 anos, na presença de fatores de risco. Pacientes cirúrgicos com menos de 40 anos sem fatores de risco são considerados como de baixo risco para TEV. Entre os pacientes clínicos, está indicada tromboprofilaxia para aqueles com idade maior ou igual a 40 anos e de mobilidade reduzida, na presença de fatores de risco. As tabelas 1 e 2 detalham os fatores de risco para TEV e a conduta indicada em cada caso.

Foi considerada conduta adequada a prescrição de medicação anticoagulante na dose e situação corretas para cada caso ou, a não prescrição, quando não havia indicação. Todas as outras condutas foram consideradas inadequadas. Na vigência de contraindicações à profilaxia medicamentosa em pacientes com necessidade de tromboprofilaxia, foi considerada adequada a indicação de métodos mecânicos (meias elásticas de compressão gradual e compressão pneumática intermitente).

Tabela 1 - Fatores de Risco para TEV

AVC

Cateteres Centrais e Swan-Ganz

Doença Inflamatória Intestinal

Doença Respiratória Grave

Doença Reumatológica Aguda

Gravidez e Pós-Parto

História prévia de TEV

IAM

ICC Classe III ou IV

Idade maior ou igual a 55 anos

Infecção

Insuficiência Arterial Periférica

Internação em UTI

Obesidade (IMC maior ou igual a 30)

Paresia/Paralisia de MMII

Quimio/Hormonioterapia

Reposição Hormonal/ Contraceptivos

Síndrome Nefrótica

Trombofilia

Varizes/Insuficiência Venosa Crônica

Tabela 2 - Risco de TEV e conduta em pacientes internados.

\begin{tabular}{lll}
\hline Grupo & Estratificação & Conduta Indicada \\
\hline Pacientes Cirúrgicos & Baixo Risco & Deambulação precoce \\
& Risco Intermediário & HNF 5000U 2 vezes ao dia \\
& & Enoxaparina 20mg 1 vez ao dia \\
& Alto Risco & HNF 5000U 3 vezes ao dia \\
Pacientes Clínicos & Enoxaparina 40mg 1 vez ao dia \\
& Profilaxia Não-Indicada & Deambulação precoce \\
Contraindicação à Profilaxia & Profilaxia indicada & HNF 5000U 3 vezes ao dia \\
& & Enoxaparina 40mg 1 vez ao dia \\
\end{tabular}


Uma amostra de 250 pacientes foi calculada como ideal para um nível de confiança de $99,9 \%$, estimando-se o percentual de inadequação da tromboprofilaxia em torno de 30 a $50 \%$. A coleta de dados e análise estatística foram realizadas com o auxílio dos softwares Epilnfo 3.4.3 e SPSS 17.0. Análise descritiva e teste qui-quadrado foram os métodos empregados.

\section{RESULTADOS}

Dos 247 pacientes analisados, 41,7\% são do sexo feminino e $58,3 \%$ do sexo masculino. De acordo com as diretrizes da $8^{\text {a }}$ ACCP, a profilaxia foi indicada para 199 pacientes, compreendendo $72,1 \%$ dos pacientes clínicos e $86 \%$ dos cirúrgicos (Tabela 3 ). Na amostra estudada, não houve nenhum caso de TEV durante o período estudado.

Dentre os fatores de risco conhecidos, os mais prevalentes nesta amostra foram: idade maior ou igual a 55 anos, infecção, internação em UTI e câncer (Tabela 3).

A minoria dos pacientes clínicos esteve internada em UTI (18,6\%), enquanto $46 \%$ dos pacientes cirúrgicos estiveram internados nesse setor (Tabela 3).

Entre os pacientes cirúrgicos, 66\% foram considerados de alto risco para TEV, $14 \%$ de risco intermediário e $20 \%$ de baixo risco. A maior parte das intervenções foi de cirurgias oncológicas (18,6\%), ortopédicas (26,6\%) e do aparelho digestivo $(21,4 \%)$.

Tabela 3 -

Características dos pacientes incluídos nos dois grupos de estudo, local de internação, indicação de profilaxia e estratificação dos fatores de risco.

\begin{tabular}{lcc}
\hline & \multicolumn{2}{c}{ Grupo } \\
\cline { 2 - 3 } & Clínico & Cirúrgico \\
\hline Número de pacientes & 97 & 150 \\
Sexo & & \\
$\quad$ Feminino & $42,8 \%$ & $40,6 \%$ \\
$\quad$ Masculino & $57,1 \%$ & $59,3 \%$ \\
Idade (anos) & $58,6 \pm 20$ & $52,2 \pm 18$ \\
Dias de Internação & $8 \pm 7$ & $7 \pm 5$ \\
Local de Internação & & \\
$\quad$ Enfermaria & $81,4 \%$ & $54 \%$ \\
$\quad$ UTI/UCO & $18,6 \%$ & $46 \%$ \\
Profilaxia Indicada & & \\
$\quad$ Mecânica & $11,3 \%$ & $2,7 \%$ \\
$\quad$ Medicamentosa & $60,8 \%$ & $83,3 \%$ \\
Profilaxia Aplicada & $46,4 \%$ & $53,3 \%$ \\
Fatores de Risco & & \\
$\quad$ Idade > 55 anos & $53,6 \%$ & $45,3 \%$ \\
Infecção & $48,4 \%$ & $19,3 \%$ \\
Internação em UTI & $18,5 \%$ & $46 \%$ \\
Câncer & $9,2 \%$ & $19,3 \%$ \\
\hline
\end{tabular}

A figura 1 evidencia a porcentagem de condutas adequadas e inadequadas na profilaxia de TEV. Em $47 \%$ das ocasiões, a conduta foi considerada inadequada, sendo a não realização de profilaxia a maior causa de inadequação. Dosagem errada e duração inadequada do anticoagulante também foram encontradas.

Não houve diferença estatisticamente significante na porcentagem de inadequação entre pacientes cirúrgicos e clínicos (44 vs. $48 \%$, respectivamente; $p=0,572$ ), assim como entre pacientes internados em enfermaria e em UTI (49 vs. $43 \%$, respectivamente; $p=$ $0,349)$.

Entre as intervenções cirúrgicas, foram encontradas taxas de inadequação de $57 \%$ para cirurgias oncológicas, 50\% para cirurgias do aparelho digestivo e


Figura 1 - Percentual de condutas adequadas e inadequadas na profilaxia de TEV (A), com detalhamento em $B$ das causas de inadequação na amostra estudada. 


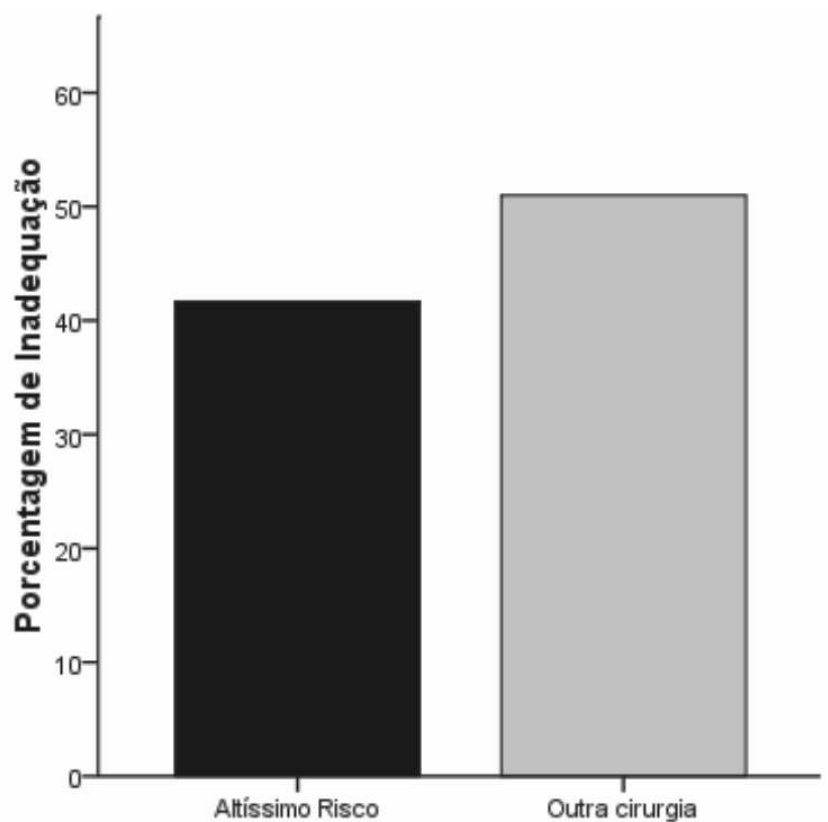

Figura 2 - $\quad$ Percentual de Inadequação da conduta, segundo classificação da cirurgia.

35\% para cirurgias ortopédicas, sem diferença estatisticamente significante entre elas. Não houve diferença também quando comparadas operações de altíssimo risco com as demais operações (41,7 vs. $51 \%$, respectivamente; $p=$ 0,287 (Figura 2).

Houve correlação estatisticamente significante entre quantidade de fatores de risco para TEV e percentual de condutas inadequadas, ao considerar-se somente casos nos quais foi indicada profilaxia para TEV. As taxas de inadequação da conduta foram maiores nos grupos de pacientes com menos fatores de risco (Figura 3).

Ainda considerando apenas casos com indicação para profilaxia, a porcentagem de inadequação da conduta também esteve correlacionada negativamente com a faixa etária dos pacientes e a duração da internação (Figuras 4 e 5).

\section{DISCUSSÃO}

Neste estudo foi encontrado percentual de adequação da profilaxia longe do ideal. Em pacientes clínicos, considerou-se a qualidade da profilaxia como adequada em 55,6\% dos casos; no grupo de cirúrgicos, 52\%. Quanto ao índice geral de adequação, esse foi de $53 \%$. Em relação ao percentual restante de condutas, consideradas inadequadas (47\%), a maior parte (33\%) foi devido a não realização da profilaxia. $11 \%$ foi devido a dose equivocada e $4 \%$ a duração errada. Dados alarmantes apontam para percentuais de inadequações consideravelmente elevados para situações que se relacionam com

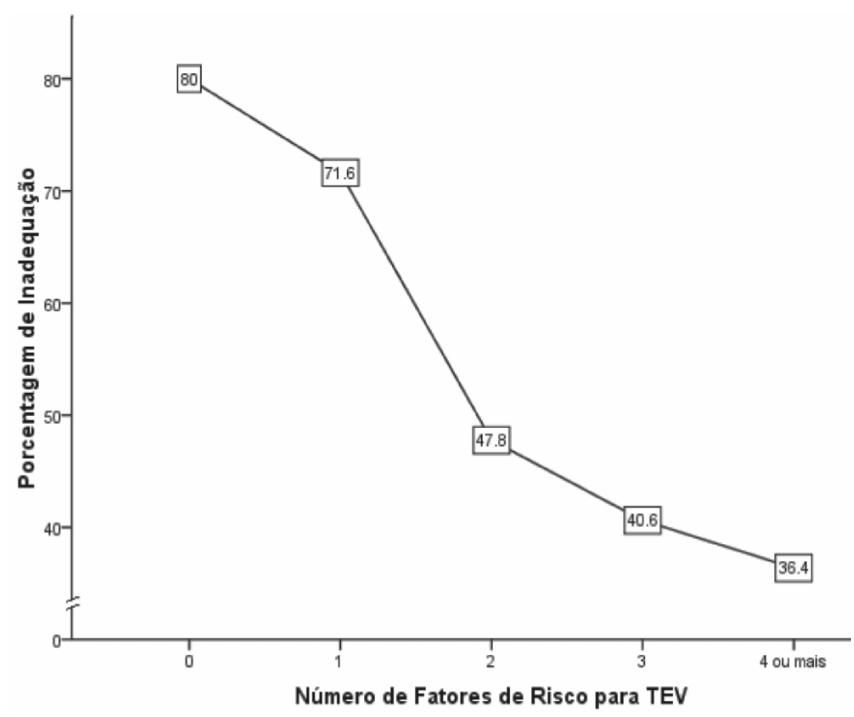

Figura 3 - $\quad$ Percentual de Inadequação da profilaxia, segundo número de fatores de risco para TEV. $\left({ }^{*} p<0,05\right.$ vs. demais pontos do gráfico)

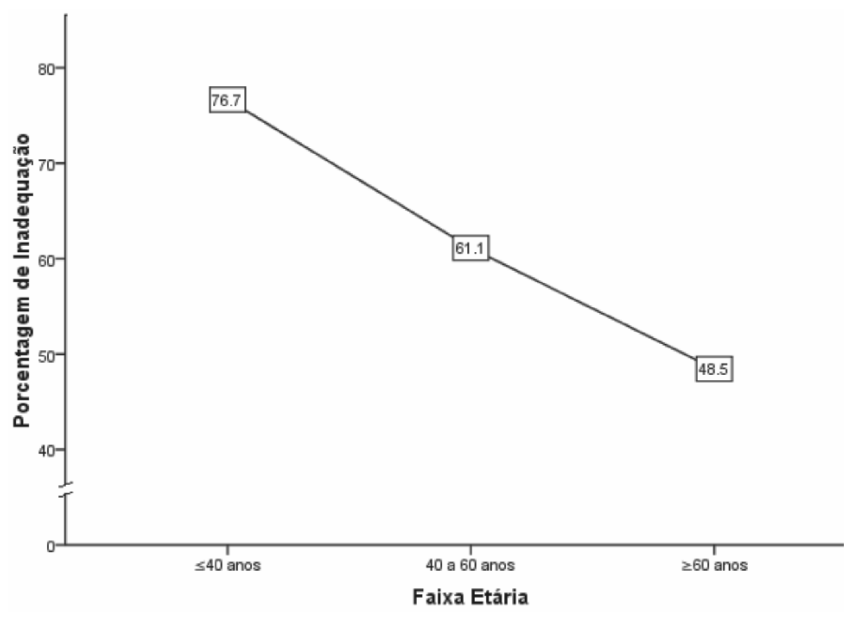

Figura 4 - Percentual de Inadequação da profilaxia, segundo faixa etária dos pacientes. ( ${ }^{*} p=0,05$ vs. demais pontos do gráfico)

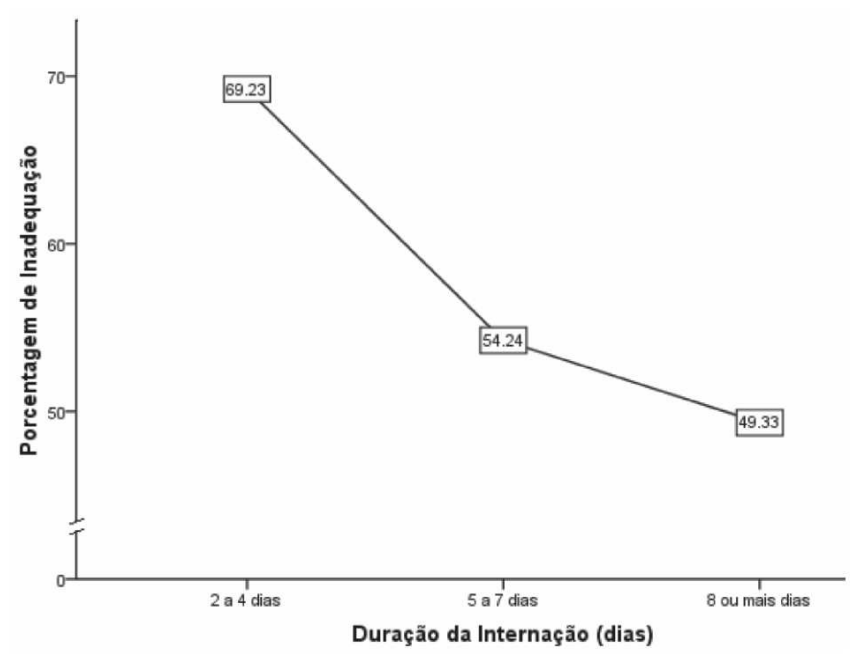

Figura 5 - $\quad$ Percentual de Inadequação da profilaxia, segundo duração da internação dos pacientes. ( ${ }^{*} p<0,05$ vs. demais pontos do gráfico) 
alto risco de TEV, como cirurgia oncológica (57\%) e internação em UTI/UCO (43\%). Os procedimentos cirúrgicos considerados de "altíssimo risco" também corresponderam a grande número de casos de tromboprofilaxia inadequada ( $41,7 \%$ das ocasiões), sem diferença estatística em relação aos demais tipos de operação, uma situação inaceitável, na medida em que trata-se de situações freqüentes e de risco bem estabelecido na literatura.

A profilaxia da TEV é subutilizada, tanto na realidade de saúde dos países desenvolvido, quanto na dos países emergentes. Nas pesquisas de Goldhaber \& Tapson ${ }^{20}$, dos 2726 pacientes com diagnóstico de Trombose Venosa Profunda em regime de internação hospitalar, somente 1147 (42\%) receberam profilaxia nos 30 dias que antecederam o diagnóstico. Trabalho publicado em Porto Alegre - RS, por Pitta et al. ${ }^{4}$, aponta para uma realidade também preocupante: dos 198 pacientes com indicação para receber profilaxia medicamentosa e/ou física, apenas $6 \%$ dos pacientes de alto risco e $18 \%$ daqueles de risco moderado receberam profilaxia.

Estudos envolvendo pacientes críticos apontam que a heparina é o método de maior eficácia na prevenção do TEV. Em pacientes de alto risco para a ocorrência de TEV, a heparina de baixo peso molecular (HBPM) parece ser mais eficiente do que a heparina não fracionada $(\mathrm{HNF})^{21,22}$. Ademais, a administração de HBPM é mais cômoda - apenas uma aplicação diária, sem necessidade de controle laboratorial do Tempo de Tromboplastina Parcial Ativada) - TTPA - e mais segura (menor ocorrência de plaquetopenia induzida por heparina $)^{23}$. Notou-se neste estudo uma freqüência reduzida, se comparada à literatura, de trombocitopenia associada ao uso de heparina (dados não mostrados em tabelas). Talvez isso se deva à relativa falta de procura ativa por essa complicação; no entanto, é mais provável que se deva ao perfil preferencial de uso de HBPM no hospital analisado.

Foi encontrada, nesta amostra, correlação estatisticamente significante entre o percentual de condutas inadequadas e o número de fatores de risco, o que aponta para uma provável desatenção do médico-assistente em cenários como o de pacientes sem fatores de risco, porém, com indicação de profilaxia para TEV (como por exemplo no caso de intervenções cirúrgicas de altíssimo risco) ou pacientes com poucos fatores de risco para TEV. De maneira inversa a Deheinzelin et al. ${ }^{19}$, que identificaram uma menor taxa de inadequação da tromboprofilaxia entre pacientes jovens (menor que 40 anos), observamos no estudo uma maior quantidade de condutas impróprias entre os pacientes mais jovens, resultado que pode ser devido à menor freqüência de fatores de risco entre os pacientes desta faixa.
Em relação à quantificação dos casos de tromboembolismo venoso hospitalar, este estudo não teria poder de determinar a prevalência real da doença. $\mathrm{O}$ estabelecimento real do TEV exige, em virtude de grande quantidade de casos oligo ou assintomáticos, protocolos mais exigentes direcionados especificamente a este fim.

É interessante relatar que, nos últimos anos, com a preocupação sobre custos de internação, as altas hospitalares são cada vez mais precoces, fazendo com que muitos casos de TEV ocorram no domicílio, após a alta hospitalar. Neste ínterim, a profilaxia deve ser mantida após a alta hospitalar. Evidencia-se, então, mais uma limitação deste estudo, no qual não se obteve contato com a prescrição de alta do paciente e, mesmo se assim o tivesse, não haveria o controle da correta utilização por parte dos pacientes no âmbito domiciliar.

Permanecem alguns questionamentos quanto a esta baixa aplicação de métodos preventivos para TEV. No caso dos pacientes cirúrgicos, seria a preocupação dos profissionais de saúde quanto ao risco de sangramento, embora já se tenha demonstrado que o uso de substâncias profiláticas não aumentou o seu risco? Seria o desconhecimento dos especialistas da real incidência de TVP e TEP NÂO) em seus pacientes? Seria, ainda, o custo financeiro que a profilaxia pode gerar, apesar de Bergqvist et al. ${ }^{24}$ mostrarem claramente que sua utilização apresenta custobenefício positivo? Ou seriam as dúvidas a respeito da classificação em grupos de risco e a adequada indicação para cada grupo?

Anderson et al. ${ }^{25}$ relataram um aumento no uso da profilaxia de $29 \%$ para $52 \%$ em pacientes hospitalizados com risco importante de desenvolvimento de trombose venosa após a introdução de estratégias educacionais com o objetivo de alertar os profissionais para a importância da prevalência do tromboembolismo. Programas educacionais sobre a adequada profilaxia da trombose venosa para profissionais da área da saúde são de extrema importância e aparentam ser um caminho para otimizar a profilaxia do TEV em pacientes internados. Outra medida importante e acessível seria anexar um protocolo em cada prontuário, seja informatizado ou não, de modo a atentar os médicos a este $\mathrm{fim}^{4,26,27}$

Os métodos que delinearam este estudo, com padronização de fatores de risco, categorização de grupos de risco e recomendação de profilaxia de TEV se mostram adequados para aprimorar os conhecimentos sobre a doença e sobre a classificação de risco tromboembólico venoso, além de otimizarem a aplicação de medidas preventivas em ambiente hospitalar, podendo, portanto, serem adotadas como forma de reverter a realidade observada. 


\section{A B S S T R A C T}

Objective: This study aimed at assessing the adequacy of thromboprophylaxis in a high complexity hospital in Vitória - ES, analysing the possible predictors of inadequate prescriptions and/or procedures. Methods: A cross-sectional study was carried out through prompt-book analysis. The included patients were hospitalized in 2007 and had their Venous thromboembolism (VTE) risk stratified using the 8th Edition of the American College of Chest Physicians (ACCP) Evidence-Based Clinical Practice Guidelines. The thromboprophylaxis adequacy was determined through a comparison between the adopted prescriptions and/or procedures and the guideline recommendations. Epilnfo 3.4.3 and SPSS 13.0 were the software applications used. Results: In 47\% of the patients the thromboprophylaxis was inadequate, being the non-prescription of the indicated medication the major reason (33\%). There was no statistically significant difference in inadequate tromboprophylaxis rate between clinical and surgical patients, or ward and Intensive care unit (ICU) ones. An inverse relationship was observed between the inadequate tromboprophylaxis rate and the number of VTE risk factors presented by the patients, as well as their age, and the length of hospital stay $(p<0,05)$. Conclusion: The results show alarming levels of thromboprophylaxis inadequacy, inacceptable in these times of well-established published guidelines. Therefore, a continuing education program should be implanted for all the assistance team.

Key words: Venous thromboembolism. Prophylaxis. Risk factors. Heparin.

\section{REFERENCIAS}

1. Francis CW. Prophylaxis for thromboembolism in hospitalized medical patients. N Engl J Med. 2007; 356(14): 1438-44

2. Geerts WH, Bergqvist D, Pineo GF, Heit JA, Samama CM, Lassen MR et al. Prevention of venous thromboembolism: American College of Chest Physicians Evidence-Based Clinical Practice Guidelines (8 $8^{\text {th }}$ edition). Chest. 2008; 133(6. Suppl): 381S-453S.

3. National Institute for Health and Clinical Excellence. Reducing the risk of venous thromboembolism (deep vein thrombosis and pulmonary embolism) in inpatients undergoing surgery. NICE clinical guideline No. 46:1-160. Available from: http://www.nice.org.uk/ CG046

4. Leite TL, Melo CFL, Calheiros GA, Silva MDC, Pitta GBB. Avaliação da utilização de profilaxia da trombose venosa profunda em um hospital escola. J Vasc Bras. 2007; 6(4): 344-351.

5. Ribeiro MA, Netto PG, Lage SG. Desafios na profilaxia do tromboembolismo venoso: abordagem do paciente crítico. Rev Bras Ter Intensiva 2006: 18(3): 316-9.

6. Franco RM, Simezo V, Bortoleti RR, Braga EL, Abrão AR, Linardi F, Costa JA. Profilaxia para tromboembolismo venoso em um hospital de ensino. J Vasc Bras. 2006: 5(2): 131-8.

7. Caiafa JS, Bastos M. Programa de profilaxia do tromboembolismo venoso do Hospital Naval Marcílio Dias: um modelo de educação continuada. J Vasc Bras. 2002; 1(2): 103-12.

8. Geerts WH, Heit JA, Claggett GP, Pineo GF, Colwell CW, Anderson FA et al. Prevention of deep venous thromboembolism. Chest. 2001; 119 Suppl 1: 132S-75S.

9. Kakkar AK, Davidson BL, Haas SK: The Investigators Against Thromboembolism (INATE) Core Group. Compliance with recommended prophylaxis for venous thromboembolism: improving the use and rate of uptake of clinical practice guidelines. J Thromb Haemost 2004; 2(2): 221-7.

10. Sullivan SD, Kahn SR, Davidson BL, Borris L, Bossuyt P, Raskob G. Measuring the outcomes and pharmacoeconomic consequences of venous thromboembolism prophylaxis in major orthopaedic surgery. Pharmacoeconomics. 2003; 21(7):477-96.

11. Caprini JA, Botteman MF, Stephens JM, Nadipelli V, Ewing MM, Brandt $S$ et al. Economic burden of long-term complications of deep vein thrombosis after total hip replacement surgery in the United States. Value Health. 2003; 6(1): 59-74.

12. Avorn J, Winkelmayer WC. Comparing the costs, risks, and benefits of competing strategies for the primary prevention of venous thromboembolism. Circulation. 2004; 110 (24 Suppl 1):IV25-32.

13. Cohen AT, Davidson BL, Gallus AS, Lassen MR, Prins MH, Tomkowski $W$ et al. Efficacy and safety of fondaparinux for the prevention of venous thromboembolism in older acute medical patients: randomized placebo controlled trial. BMJ. 2006; 332(7537): 325 9. Epub 2006 Jan 26.

14. Samama MM, Cohen AT, Darmon JY, Desjardins L, Eldor A, Janbon $C$ et al. A comparison of enoxaparin with placebo for the prevention of venous thromboembolism in acutely ill medical patients. Prophylaxis in Medical Patients with Enoxaparin Study Group. N Engl J Med. 1999; 341(11): 793-800.

15. Leizorovicz A, Cohen AT, Turpie AG, Olsson CG, Vaitkus PT, Goldhaber SZ; PREVENT Medical Thromboprophylaxis Study Group. Randomized, placebo-controlled trial of dalteparin for the prevention of venous thromboembolism in acutely ill medical patients. Circulation. 2004; 110(7): 874-9. Epub 2004 Aug 2.

16. Ellis $\mathrm{MH}$, Elis A. Perioperative venous thromboembolism prophylaxis in Israel: a survey of academic surgical departments. Eur J Haematol 2004; 73(2): 104-8.

17. National Institute of Clinical Studies. The prevalence of chemoprophylaxis in surgical and medical cases at high risk of venous thromboembolism. Melbourne, Australia: School of Population Health, University of Western Australia, 2005; 127.

18. Rashid ST, Thursz MR, Razvi NA, Voller R, Orchard T, Rashid ST, Shlebak AA. Venous thromboprophylaxis in UK medical inpatients. J R Soc Med. 2005; 98(11): 507-12.

19. Deheinzelin D, Braga AL, Martins LC, Martins MA, Hernandez A, Yoshida WB, et al. Incorrect use of thromboprophylaxis for venous thromboembolism in medical and surgical patients: results of a multicentric, observational and cross-sectional study in Brazil. Thromb Haemost. 2006; 4(6): 1266-70.

20. Goldhaber SZ, Tapson VF; DVT FREE Steering Commitee. A prospective registry of 5,451 patients with ultrasound-confirmed deep vein thrombosis. Am J Cardiol. 2004; 93(2): 259-62.

21. Prevention of venous thromboembolism. International Consensus Statement (guidelines according to scientific evidence). Int Angiol. 1997; 16(1): 3-38.

22. Bergmann JF, Neuhart E. A multicenter randomized double-blind study of enoxaparin compared with unfractionated heparin in the prevention of venous thromboembolic disease in elderly in-patients bedridden for an acute medical illness. Thromb Haemost. 1996; 76(4): 529-34

23. Martel N, Lee J, Wells PS. Risk for heparin-induced thrombocytopenia with unfractionated and low-molecular-weight heparin thromboprophylaxis: a meta-analysis. Blood. 2005; 106(8): 2710-5. Epub 2005 Jun 28.

24. Bergqvist $D$, Lindgren $B$, Mätzsch T. Comparison of the cost of preventing postoperative deep vein thrombosis with either unfractionated or low molecular weight heparin. Br J Surg. 1996; 83(11):1548-52. 
25. Anderson FA Jr, Wheeler HB, Goldberg RJ, Hosmer DW, Forcier A, Patwardhan NA. Physician practices in the prevention of venous thomboembolism. Ann Intern Med. 1991; 115(8): 591-5.

26. Engelhorn ALV, Garcia ACF, Cassou AF, Birckholz L, Engelhorn CA. Profilaxia da trombose venosa profunda: estudo epidemiológico em um hospital escola. J Vasc Bras. 2002; 1(2): 97-102.

27. Stinnett JM, Pendleton R, Skordos L, Wheeler M, Rodgers GM Venous thromboembolism prophylaxis in medically ill patients and the development of strategies to improve prophylaxis rates. Am J Hematol. 2005; 78(3): 167-72.
Recebido em 02/03/2009

Aceito para publicação em 29/05/2009

Confliot de interesse: nenhum

Fonte de financiamento: nenhuma

\section{Como citar este artigo:}

Carneiro JLA, Targueta GP, Marino LO. Avaliação da profilaxia do tromboembolismo venoso em hospital de grande porte. Rev Col Bras Cir. [periódico na Internet] 2010; 37(3). Disponível em URL: http:// www.scielo.br/rcbc

Endereço para correspondência:

João Luiz de Aquino Carneiro

E-mail: mouton.vix@terra.com.br 